$$
\text { PART IV }
$$

\title{
ABSOLUTE MAGNITUDE DETERMINATIONS \\ FROM HYDROGEN-LINE PHOTOMETRY
}




\title{
ABSOLUTE MAGNITUDE DETERMINATIONS FROM HYDROGEN-LINE PHOTOMETRY
}

\author{
D. L. CR AWFORD \\ Kitt Peak National Observatory*, U.S.A.
}

\begin{abstract}
The history and status of Balmer hydrogen-line photometry are briefly reviewed, and problems associated with calibrations in general are commented on.

The calibration work at Kitt Peak is described in some detail for B, A, and F type stars. The data used, the determination of intrinsic colours and colour excesses, photometric classification, and the relation of our indices to other people's are reviewed. With this background, the procedure used in establishing the absolute magnitude $-\mathrm{H} \beta$ calibration is given, and the preliminary calibration is presented. Finally, the work remaining to be done before the calibration is final is noted, and a comparison is given to Blaauw's zero-age main sequence calibration.
\end{abstract}

\section{History}

The history of the use of. Balmer-line strengths for the determination of stellar luminosities has been a rich one over the past $50 \mathrm{yr}$, and I cannot hope to review it all, or even mention it all, here today. I will content myself, and I hope you, by briefly noting some of the past work that I feel has been particularly influential, by summarizing the current status of Balmer hydrogen-line work, and by describing in some depth the work I am most acquainted with: my own calibration efforts with the $\beta$ and $u v b y$ systems.

Pre-1950, the major work was by Lindblad (1922, 1925, 1926), Anger $(1931,1932)$, Öhman (1935), and Williams (1936), who measured Balmer lines on spectral plates and discussed the relation of the derived hydrogen-line strengths to stellar luminosities. Data for stars in clusters were especially useful in such discussions, as the relative luminosities of the stars were well known.

Later, Petrie and his collaborators at Victoria (Petrie, 1950, 1953, 1956, 1965; Petrie and Maunsell, 1950; Petrie and Moyls, 1956) developed and applied the photographic technique to determine hydrogen-line equivalent widths from spectral plates for B-type stars. The technique and calibrations are reviewed and discussed in depth by Petrie (1965) in a paper giving the revision to his earlier calibration. In a paper later today, Crampton will summarize the Victoria system as it now stands.

Others who lately have been in photographic hydrogen-line efforts are Hack (1953), Kopylov (1958), Sinnerstad (1954, 1961a, b), Beer (1961), and Furenlid (1971).

Soon after Petrie's extensive effort, photoelectric techniques to measure a parameter related to hydrogen-line strengths were developed and used by Strömgren (1951, 1952, 1956a, b, 1958), Crawford (1958), Hoag (1965), Bappu et al. (1962), Johnson and Iriarte (1958), Beer (1964), Andrews (1968), Graham (1967), and others. Absolute magnitude calibrations of the $\beta$ index, that we use, have been given

* Operated by the Association of Universities for Research in Astronomy, Inc., under contract with the National Science Foundation. 
by Hardie and Crawford (1961), Fernie (1965), and Crawford (1970), but were based on considerably less material than the calibration that I'll present later in this paper.

Strömgren (1963) gave an excellent summary of the Balmer line work to that time, and he (Strömgren, 1966) again reviewed the status of hydrogen-line spectral-classification work. Both these review papers also discussed other narrow-band photometric classifications and calibration work.

\section{Some Problems}

Calibration of anything observed vs anything more-or-less physical for B-type stars is not an easy task, as the authors above have clearly shown.

What we'd really like to have are observed parameters, easy to measure for bright and faint stars, internally accurate, free of systematic effects, that are closely (even linearly) related to a physical parameter of the stars, very sensitive to the physical parameter (that is, giving a large range of the observed parameter), and completely free of the influence of any other physical parameter. Nature has not allowed any such situation to exist, and the best we can do is try to obtain parameters that will do as good a job as possible, so that we can use them with some confidence in our astronomical research. Sometimes we can measure an extra parameter, which, while useful in itself, will also allow us to correct one of the main parameters for an undesirable side effect.

An obvious example: We measure a colour index such as $(B-V)$, which is well related to effective temperature. Side effects are interstellar reddening, abundance effects, and even rotation velocity, magnetic field, and binary star effects. Any extra parameter to help correct for these side effects, such as $(U-B)$, has side effects of its own. And so it goes. Quite difficult.

In many cases, theory can help us observers quite a lot. We would be lost, or at the least inefficient, without theory to guide us. However, we must be extremely careful (as Morgan has often pointed out) not to force-fit or to let pre-conceived ideas mess us up. We are measuring observed parameters, and these we relate, or calibrate, to physical parameters. In my opinion, this philosophy is one of the keys that has led to the success of the MK system. It is a network (or two observed 'parameters') that 'classifies' or describes a star's spectrum. It is, in principle, even independent of the spectral dispersion used, for it is defined by standard stars. The observed parameters are then calibrated in terms of temperature and absolute magnitude. In fact, they have been re-calibrated many times. Such methods and philosophy are certainly valid for the best and most useful photometric systems too, I believe.

Enough of philosophy, let me now list some of the specific problems we have to watch out for, or allow for, in hydrogen-line photometry:

(a) accurate and precise standard systems, equipment, and techniques are needed to insure an accurate parameter free of systematic error,

(b) interstellar reddening corrections,

(c) rotational velocity effects, 
(d) peculiar stars (however peculiar is defined),

(e) binaries,

(f) cluster membership,

(g) emission line stars, and

(h) accurate and precise absolute magnitudes to calibrate the observed parameter against.

\section{The Kitt Peak Work}

With this brief background, let me now go on to a detailed description of the work at Kitt Peak. The final calibrations are not quite finished, but little yet remains to be done, and I hope to finish it by the end of this year. The preliminary results presented today should differ little from the final ones.

\section{A. THE DATA}

Most of the discussion to follow will be devoted to parameters of (a) the uvby system, defined by Crawford and Barnes (1970c), that was originated by Strömgren and developed by him and by us at Kitt Peak, and (b) the $\beta$ system defined by Crawford and Mander (1966).

The parameters used are:

$V$ - an apparent magnitude, on the same system as the $V$ of the $U B V$ system;

$(b-y)-$ a colour index, freer of line blanketing effects than $(B-V)$ of the $U B V$ system;

$(u-b)$ - a colour index;

$m_{1}$ - a colour difference, related to blanketing in the $4100 \AA$ region;

$c_{1}$ - a colour difference, related to the Balmer discontinuity;

$\beta$ - the hydrogen line-strength parameter.

These parameters are discussed in the standard-system papers mentioned above, and by Strömgren $(1963,1966)$.

We also use $U B V$ and $\mathrm{MK}$ data taken from the literature, especially for comparison purposes. Not all the uvby or $\beta$ data are our own: some have been taken from the literature and some have been kindly given to me in advance of publication. I wish to thank very much those who have supplied their unpublished data to me.

Data for the following stars have been used:

(a) northern hemisphere O-B5 stars brighter than $V=6.5$, Crawford et al. (1971b);

(b) southern hemisphere $\mathrm{O}$ to $\mathrm{G} 0$ stars brighter than $V=5.0$, Crawford et al. (1970);

(c) southern hemisphere O-B5 stars $V=5.0$ to 6.5, Crawford et al. (1971a);

(d) northern hemisphere A2-G0 stars brighter than $V=6.5$, Strömgren and Perry (1965) and Crawford et al. (1966);

(e) northern hemisphere A0-type stars brighter than $V=6.5$, Crawford et al. (1972);

(f) northern hemisphere B8- and B9-type stars, Crawford et al. (unpublished);

(g) northern hemisphere O-type stars fainter than $V=6.5$, Crawford and Golson (unpublished); 
and for the following clusters and associations:

(a) Hyades, Crawford and Perry (1966);

(b) Praesepe, Crawford and Barnes (1969b);

(c) Coma, Crawford and Barnes (1969a);

(d) NGC 752, Crawford and Barnes (1970a);

(e) IC 4665, Crawford and Barnes (1972);

(f) Pleiades, Crawford (unpublished);

(g) $\alpha$ Per, Crawford and Barnes (unpublished);

(h) h and $\chi$ Per, Crawford et al. (1970);

(i) NGC 6231, Crawford et al. (1971);

(j) IC 2602, Hill and Perry (1969);

(k) IC 2391, Perry and Hill (1969);

(l) Sco-Cen, Hardie and Crawford (1961), Glaspey (1971);

(m) Orion, Crawford (1958), Crawford and Barnes (1966);

(n) III Cep, Crawford and Barnes (1970b);

(o) NGC 2362, Perry (unpublished);

(p) II Per, Crawford (1958);

(q) NGC 6871, Cohen (1969);

(r) NGC 6910, Crawford and Barnes (unpublished);

(s) NGC 6913, Crawford and Barnes (unpublished);

(t) NGC 6611, Crawford and Barnes (unpublished);

(u) NGC 5460, Clariá (1971);

(v) NGC 2264, Strom et al. (1971); and

(w) NGC 2244, Heiser (unpublished).

\section{B. INTRINSIC COLOURS AND COLOUR EXCESSES}

For the A- and F-type (those stars cooler than about A2, the location of maximum hydrogen absorption), we determine the intrinsic colour from the following equation:

$$
(b-y)_{0}=a-b \beta-c \delta c_{1}-d \delta m_{1} .
$$

For A-type stars $(\beta=2.890$ to 2.720$)$, the constants used are $a=2.943, b=1.000$, $c=0.100, d=0.100$. The resulting mean error, for one star, as determined from stars within $100 \mathrm{pc}$, is $\pm 0 \mathrm{~m} .011$. This scatter includes effects of duplicity, rotation velocity, and so forth, as essentially no data were eliminated from the least squares solution. It can be seen that $\beta$ is an effective parameter in predicting intrinsic colour, for $\mathrm{c}$ and $\mathrm{d}$ are small; i.e., little luminosity or abundance effects exist.

For F-type stars $(\beta=2.720$ to 2.600$)$, the coefficient $b$ increases as we go to later types (smaller $\beta$ ), as $\beta$ begins to lose sensitivity to temperature near G0 while $(b-y)$ does not; the coefficient $c$ is 0.1 or a bit smaller; and the coefficient $d$ increases toward later spectral type, as blanketing effects become larger.

Details and limitations of the calibrations for A- and F-type stars will be discussed fully in a forthcoming paper; a summary has been given by Crawford (1970).

For the B-type stars, we have determined colour excesses, $E(U-B)$, and intrinsic 
colours $(U-B)_{0}$, for those stars with available $U B V$ data, by a procedure described by Crawford (1958). A linear reddening slope has been assumed: $E(U-B) / E(B-V)$ $=0.72$. This use of a linear slope may not be justified in all cases, but should be adequate for the discussions to follow. I prefer the use of $(U-B)_{0}$ to $(B-V)_{0}$ for B-type stars, as the former has a considerably larger range (about 4 times larger than $\left.(B-V)_{0}\right)$. I also prefer it over the parameter $Q$ (Johnson and Morgan, 1953) as it is a more natural parameter, i.e., the intrinsic colour, and is no more difficult to determine than is $Q$.

For an overall discussion of effects of interstellar reddening on the $U B V$ parameters, I believe that the investigation by Fitzgerald (1970) is the most complete.

For investigation of reddening effects on the uvby system, I have used the data noted above. Figures 1, 2, and 3 show the relation between the observed $(u-b), m_{1}$, and $c_{1}$ indices with respect to $(b-y)$ for O-type stars. Separate symbols are used in

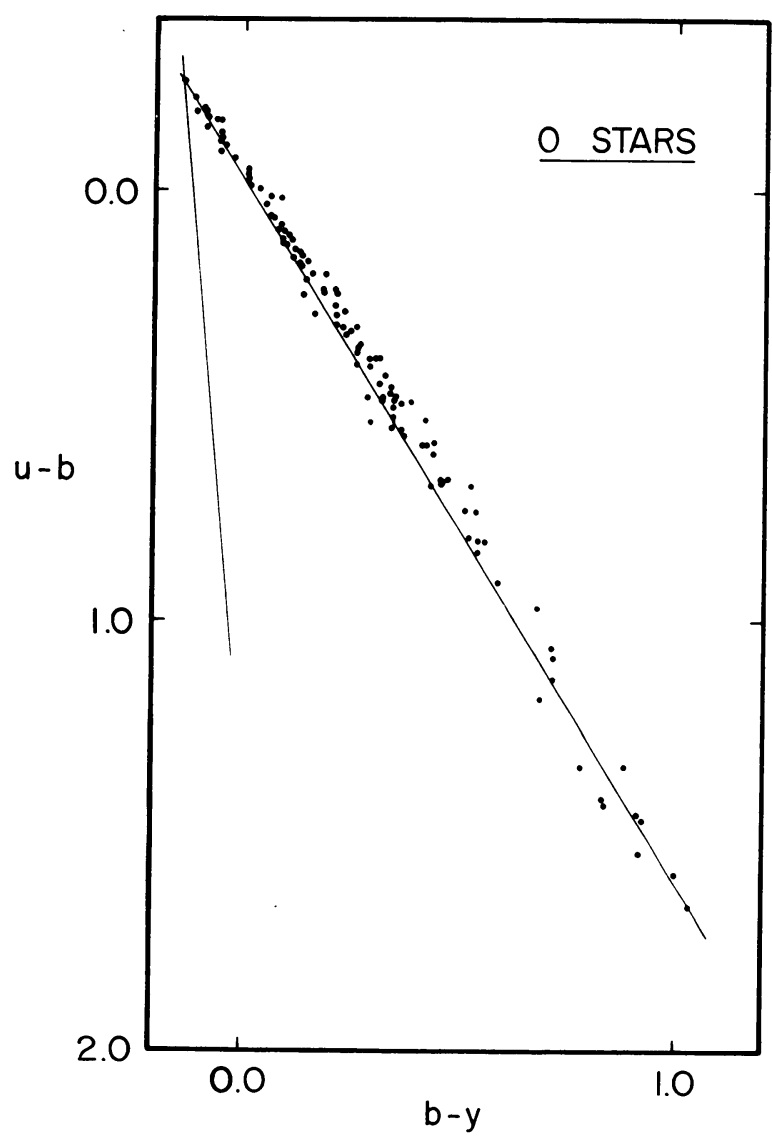

Fig. 1. The $(u-b)$ vs $(b-y)$ relation for a number of O-type stars observed from Kitt Peak. A linear line of slope 1.61 is shown through the data points. The line to the left traces the intrinsic colour line for B-type stars. 


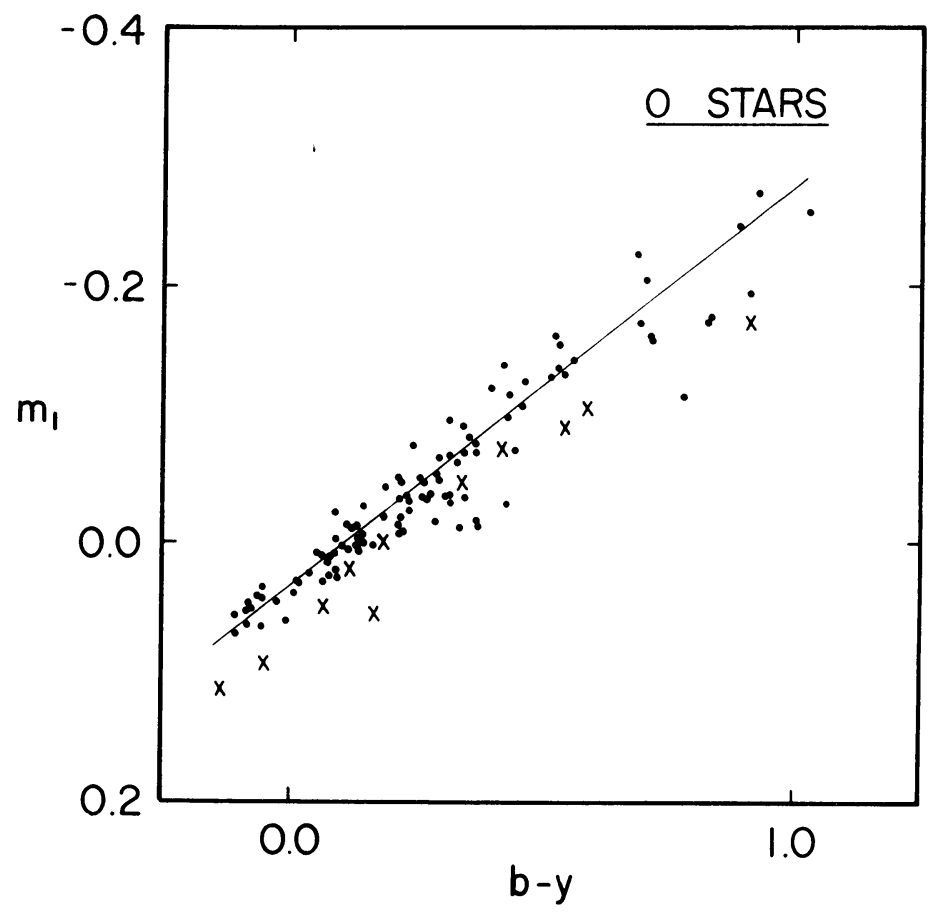

Fig. 2. The $m_{1}$ vs $(b-y)$ relation for a number of O-type stars. A reddening line, with slope near -0.3 , is shown. Crosses denote Of-type stars.

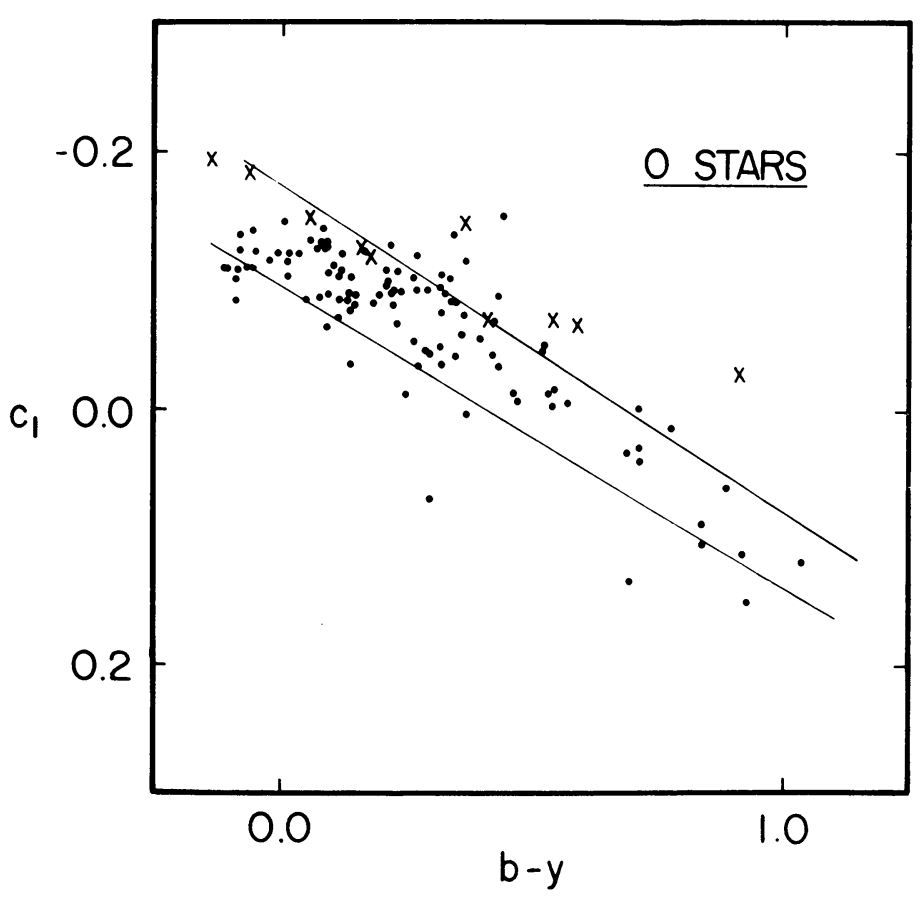

Fig. 3. The $c_{1}$ vs $(b-y)$ relation for a number of O-type stars. Two reddening lines, with slopes near 0.2 , are shown. Crosses denote Of-type stars. 
Figures 2 and 3 for the known Of type stars. Allowing for some luminosity and spectral type effects, I have derived the following reddening relations:

$$
\begin{aligned}
E(u-b) & =1.6 E(b-y), \\
E\left(m_{1}\right) & =-0.3 E(b-y), \quad \text { and } \\
E\left(c_{1}\right) & =0.2 E(b-y) .
\end{aligned}
$$

One can also derive $E(b-y)=0.73 E(B-V)$ from a plot of $(b-y)$ vs $(B-V)$ for the same stars. I have used these four reddening relations in the work described below.

Figure 4 shows the relation between the observed $c_{1}$ and $(b-y)$ indices for the brighter $\mathrm{O}$ to B5-type field stars. A well defined, left-hand envelope is evident, and it

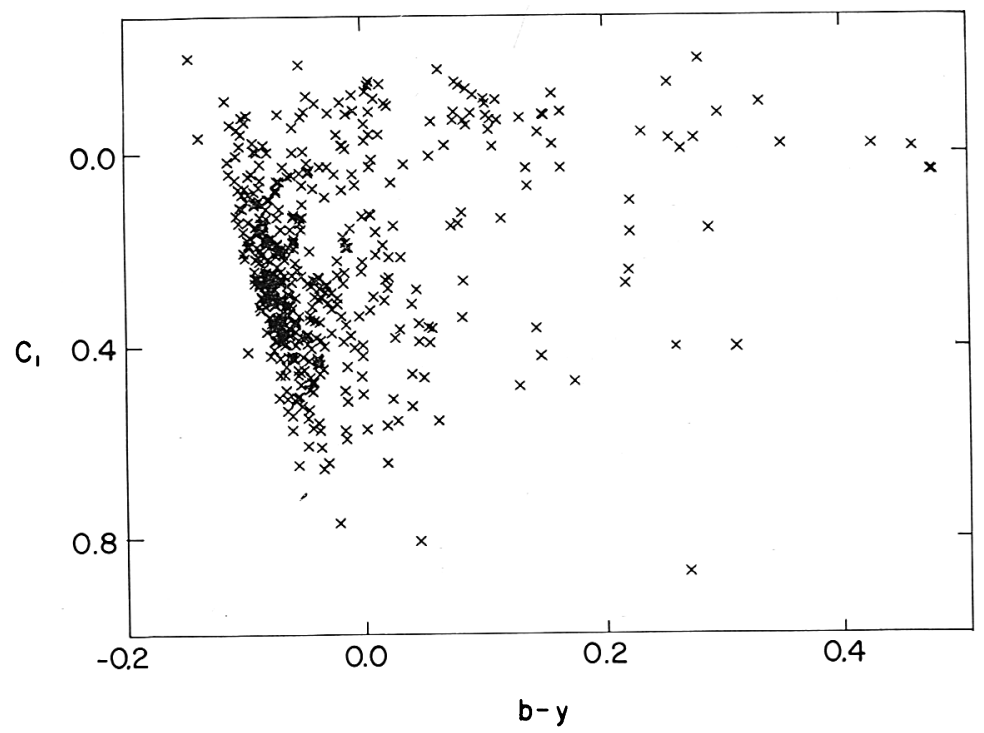

Fig. 4. The $c_{1}$ vs $(b-y)$ diagram for O-to-B5 type stars brighter than $V=6.5$. Reddening lines are nearly horizontal in the diagram, with the intrinsic colour line as the left-hand envelope of the points.

may be defined as the intrinsic colour relation (at least to a first approximation). For the preliminary calibration, I have assumed $(b-y)_{0}=-0.116+0.097 c_{0}$, where $c_{0}$ is the unreddened $c_{1}$ index. The final calibration will allow for non-linearities and for small luminosity effects evident both in the observed data for bright stars and in theoretical model atmospheres. Checks have been made by calculating colour excesses for clusters in which the reddening is either zero or essentially uniform. (See, for example, Hill and Perry (1969) and Crawford et al. (1970).)

A comparison of the reddening values so obtained with values obtained from $U B V$ data (the procedure described above) and from MK types and $(B-V)$ values shows that the agreement (where data are good) is excellent. For example, a detailed comparison of the three methods is given by Crawford et al. (1970) for the stars in the $h$ and $\chi$ Per clusters. 
In all the work to follow, we have assumed the ratio of total-to-selective absorption to be $A_{V} / E(B-V)=3.2$; i.e., $A_{V} / E(b-y)=4.3$.

C. HYDROGEN-LINE DATA, AND' PHOTOMETRIC TWO-DIMENSIONAL CLASSIFICATION FOR B-TYPE STARS

In Figure 5, I show the relation between our observed $\beta$ values and Petrie's $\mathrm{H} \gamma$ values for B-type stars with data in common. The $\mathrm{H} \gamma$ values have been taken from a recent compilation by Crampton, who has kindly sent me his data. Only a sampling of data

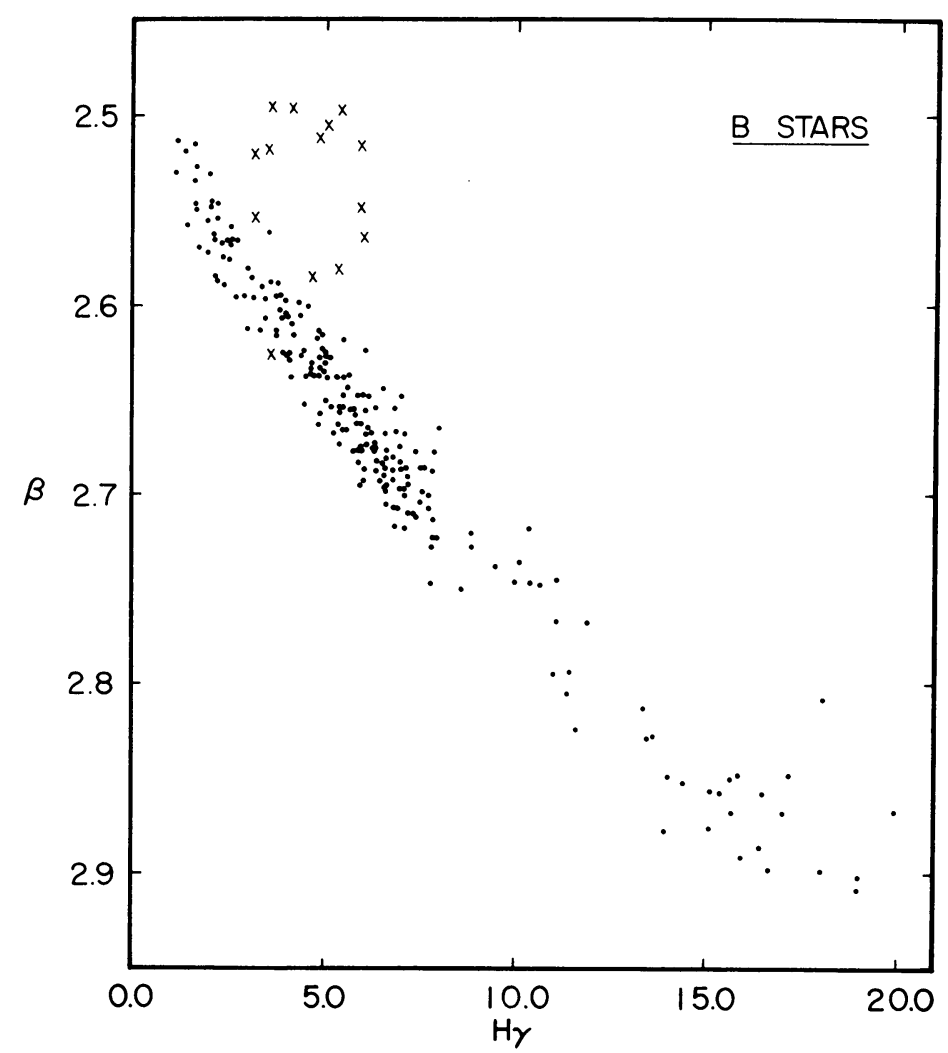

Fig. 5. The relation between the $\beta$ parameter and Petrie's $\mathrm{H} \gamma$ values, for B-type stars. Crosses denote known emission line objects; points for some such objects would lie off of the top of the figure. Only a sampling of points for late B-type stars are shown.

for the later B-type stars has been plotted, to show the trend. Emission line objects are generally well separated from the main relation. No scatter due to rotation velocity effects is apparent in the data, and the overall scatter in the relation is close to that expected from observational error alone.

In Figure 6, we show the relation between two well-observed photoelectric parameters. The data plotted are for the $\beta$ system standard stars, the $\beta$ 's taken from 


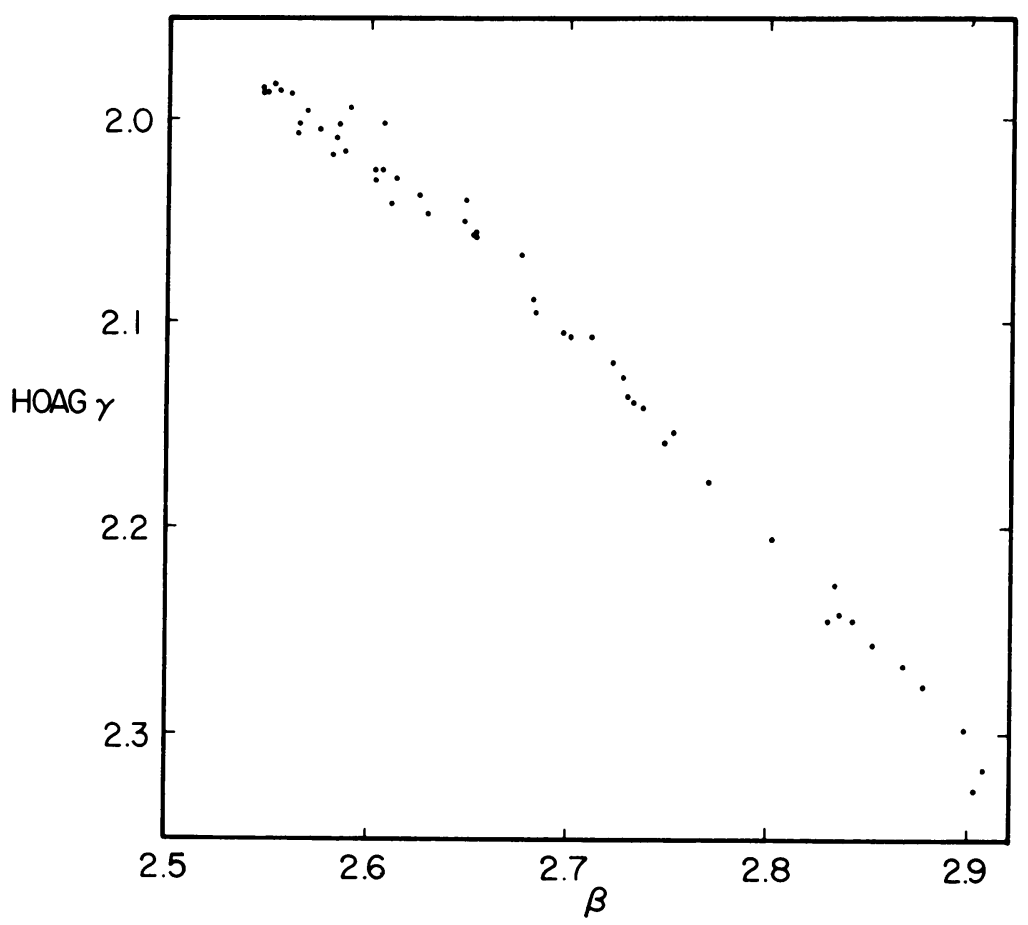

Fig. 6. The relation between the $\beta$ parameter and Hoag's photoelectric $\mathrm{H} \gamma$ parameter, for standard stars of the $\beta$ system.

Crawford and Mander (1966) and the $\mathrm{H} \gamma$ values from Hoag's unpublished $\gamma$ values for the same stars. We wish to thank him for permission to show his data. As can be seen, the scatter is quite small.

In Figure 7, we show our $\beta$ data plotted against Andrews' (1968) $\mathrm{H} \alpha$ data, for B-type stars. The points above the average relation are due to emission line stars. Again, as in the $\mathrm{H} \gamma$ vs $\beta$ plots, points for emission line stars are well separated from the main relation, and no rotational velocity effects are evident in the relation. Many supergiant stars have hydrogen emission, but the data for them lie along the main relation (upper left). The separation of such stars from main sequence emission line objects is rather easy. The turn-up for the brighter B-type stars is due to non-LTE effects, leading to emission (see Mihalas, 1972, and the references given there). The transition to emission is smooth, as theory predicts. An $\mathrm{H} \alpha$ parameter should thus be better than an $\mathrm{H} \beta$ parameter for absolute magnitude determinations for the brightest B-type stars. For later B-type stars, $\mathrm{H} \beta$ is probably better, and the two together can separate out 'emission-line stars'. Neither will work well if the emission is variable.

Photometric classification is possible with the parameters we have measured or derived above. The parameters measuring the Balmer discontinuity $\left[(U-B)_{0}, c_{0}\right]$ relate to effective temperature, the hydrogen-line parameters $[\beta, \gamma$, or $\alpha]$ relate to luminosity, or absolute magnitude. $\mathbf{A}\left(\beta, c_{0}\right)$ diagram, therefore, is rather like an HR 


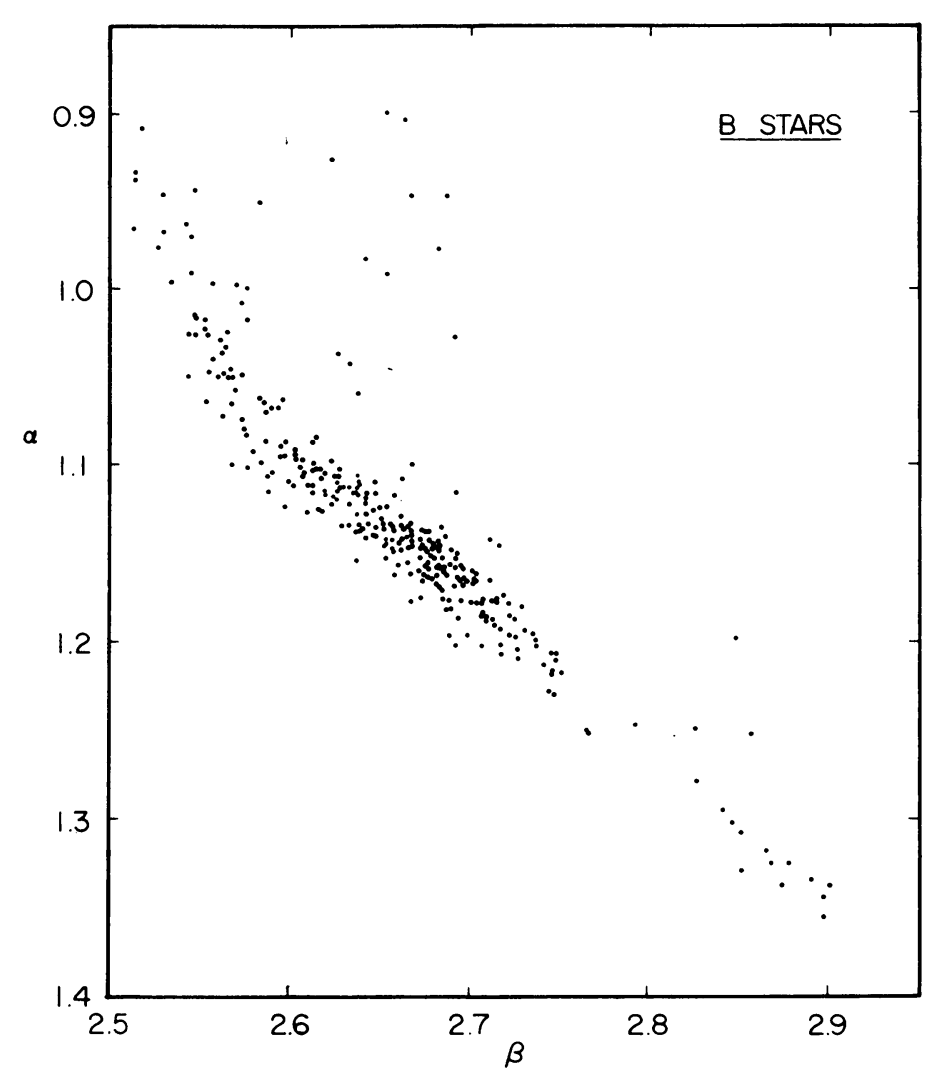

Fig. 7. The relation between the $\beta$ parameter and Andrews' photoelectric $\mathrm{H} \alpha$ parameter. Emission line objects lie above the average relation. Points for B-type supergiants lie in the upper left of the diagram. Only a sampling of points for late B-type stars are shown.

diagram, or a colour-magnitude diagram. Furthermore, 'boxes' can be drawn in such a diagram relating to $M K$ types. In fact, the relation of the photometry to the spectral types is very good; see, for example, Crawford (1958). Exceptions are usually 'peculiar' stars; for example, see Garrison (1967) and Cowley and Crawford (1971).

In Figure 8, we show the $\left(\beta, c_{0}\right)$ data for the $\mathrm{O}-\mathrm{B} 5$ stars brighter than $V=6.5$. A lower envelope is apparent. This lower boundary defines our 'zero-age main sequence' (ZAMS), and we interpret the scatter above it (except for that due to observational error!) to be due to the stars having evolved above the ZAMS and hence having greater luminosities for a given temperature. Such an interpretation is good only to a first approximation, of course, as the parameters are not ideal ones, as described in Section 2 above. In the final calibration, we will use a parameter $\delta \beta$ defined as

$$
\delta \beta=\beta \text { (ZAMS) }-\beta \text { (observed) },
$$

and relate this $\delta \beta$ to $\delta M_{V}$, where

$$
\delta M_{V}=M_{V}(\text { ZAMS })-M_{V} \text { ('observed'). }
$$




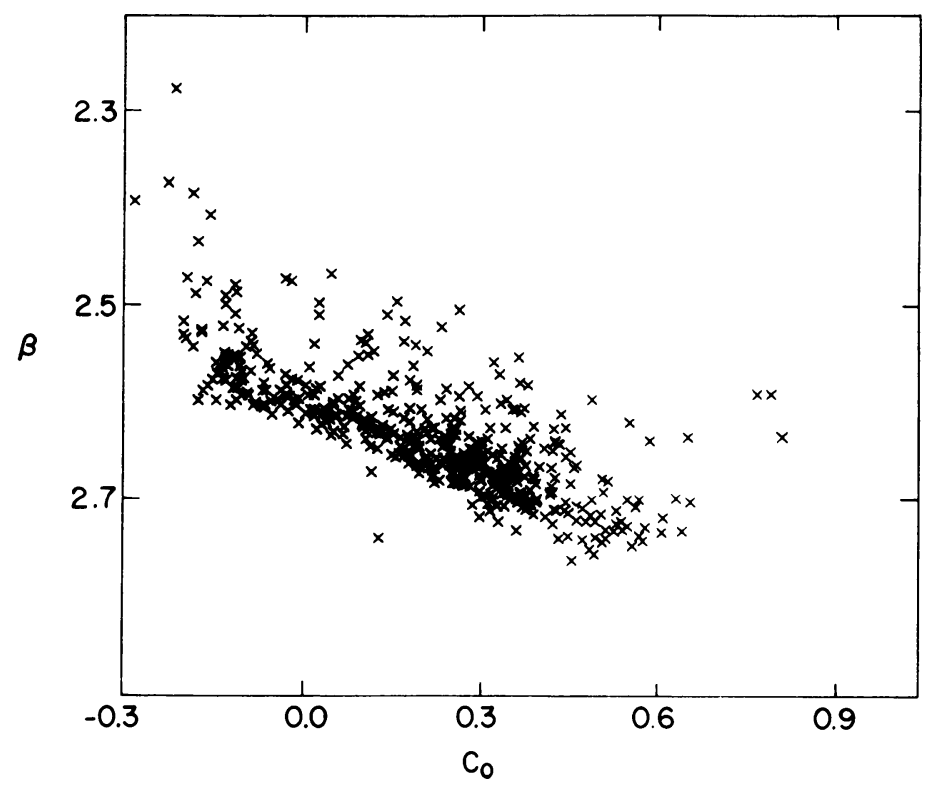

Fig. 8. The $\beta$ vs $c_{0}$ diagram for O- to B5-type stars brighter than $V=6.5$. We define the lower envelope as the 'zero-age main sequence' (ZAMS).

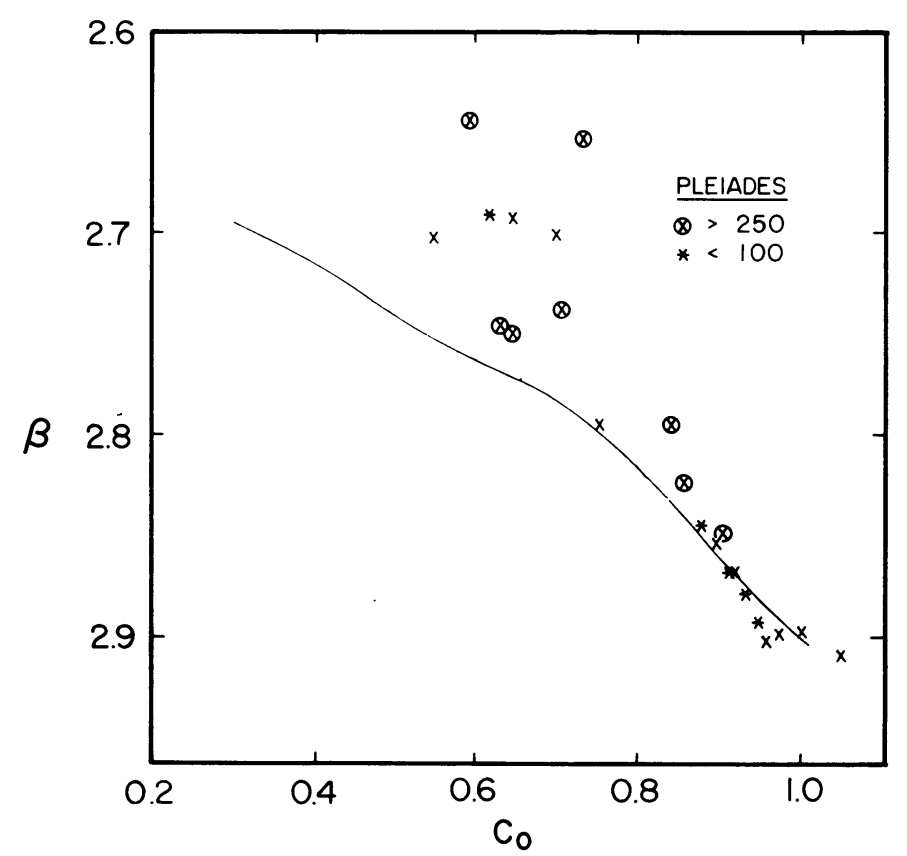

Fig. 9. The $\beta$ vs $c_{0}$ relation for B-type stars that are members of the Pleiades cluster. Different symbols denote stars with large or small $V \sin i$ values (in $\mathrm{km} \mathrm{s}^{-1}$ ). The line drawn in the diagram is the ZAMS. 
In Figure 9, we show the $\left(\beta, c_{0}\right)$ relation for the B-type stars in the Pleiades cluster. The separation of the brighter stars from the ZAMS line is evident. Little, if any, effect is noticeable due to $V \sin i$ differences.

\section{THE ABSOLUTE MAGNITUDE CALIBRATION}

We do the calibration by several distinct steps:

(1) Determine the shape of the ZAMS relation between $M_{V}$ and $\beta$ for the A and F stars, by observations in clusters. In three of the clusters used, Pleiades, $\alpha$ Per, and IC 4665, the A and F stars should be nearly unevolved; therefore, no correction for any $\delta M_{V}$ above the ZAMS has been applied. For the other clusters, a correction has been applied to those stars with significant $\delta c_{1}$ (For discussion of these corrections, see Stromgren, 1966, and Crawford, 1970.). For A-type stars $8 \delta c_{1}=\delta V_{0}$ has been added to the individual $V_{0}$ 's; for $\mathrm{F}$ stars $11 \delta c_{1}=\delta V_{0}$ was used. The $V_{0}$ vs $\beta$ relation for the stars in IC 4665 is shown in Figure 10, as an example. Diagrams for the separate clusters were overlayed, sliding the diagrams vertically along lines of equal $\beta$, and

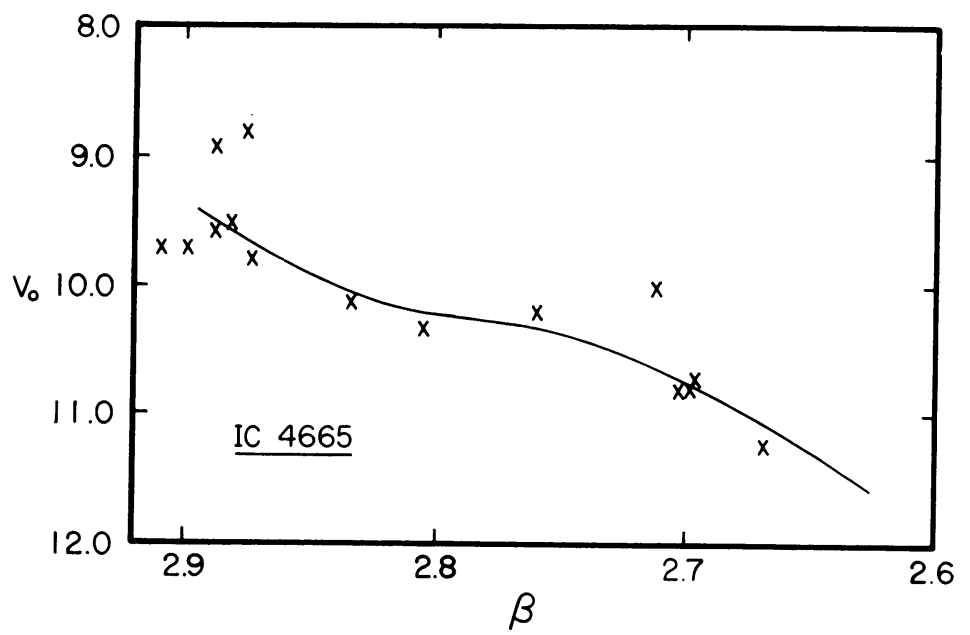

Fig. 10. The $V_{0}$ vs $\beta$ relation for A, F-type members of the cluster IC 4665 . A mean relation is drawn through the points.

a smooth mean relation best fitting the individual relations was drawn. The Hyades and Praesepe were not used in this determination.

(2) Determine the zero-point of the relation from a fit to trigonometric parallax stars. Absolute magnitudes were calculated for those stars with parallaxes greater than 0"100, in the Yale Trigonometric Parallax Catalog (Jenkins, 1963), and for those with parallaxes greater than 0.060 having large weight. The resultant absolute magnitudes were plotted in a $\left(M_{V}, \beta\right)$ diagram, and the corrections for $\delta M_{V}$ applied whenever $\delta c_{1}$ was not equal to zero. The mean slope determined above, in Step 1, was then fitted to the corrected points. The result is shown in Figure 11. It can be seen that the mean cluster slope fits the data for the parallax stars quite well. 


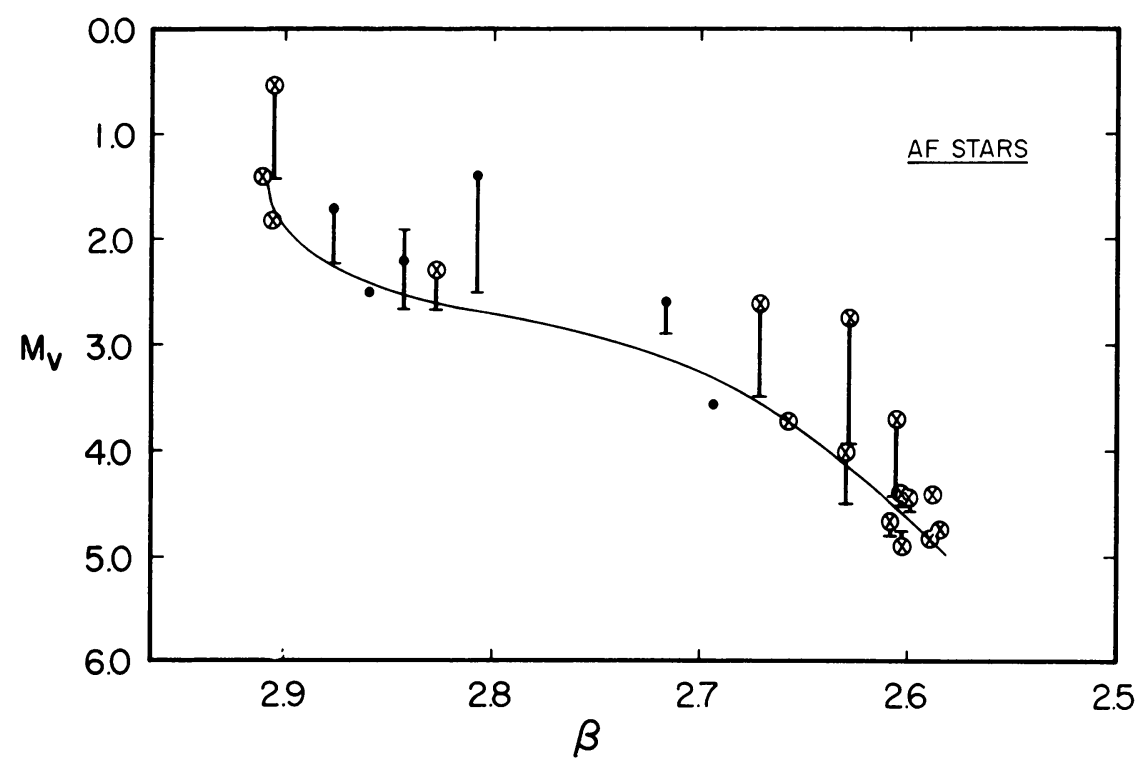

Fig. 11. The $M_{V}$ vs $\beta$ relation for A, F-type stars with large trigonometric parallaxes. The vertical line with the cross-bar shows the correction for evolutionary effects (see text). The mean relation from clusters is drawn in the figure, as best fitting the points for the parallax stars. The line, therefore, is the calibration valid for the ZAMS for the A- and F-type stars.

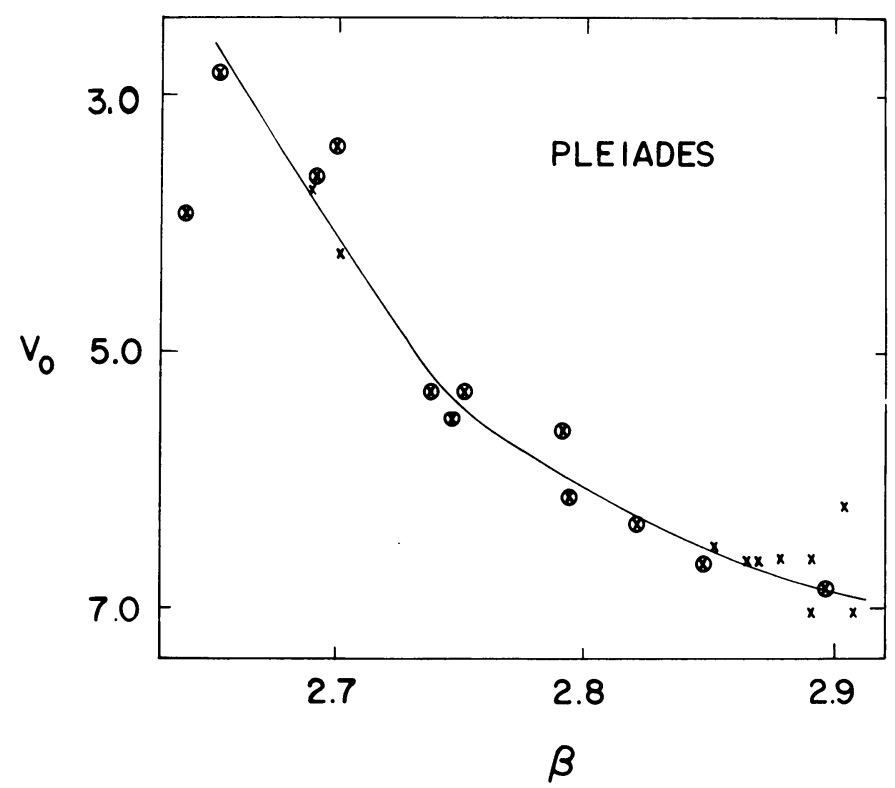

Fig. 12. The $V_{0}$ vs $\beta$ relation for B-type members of the Pleiades cluster. The crosses with circles about them denote stars with $V \sin i$ greater than $200 \mathrm{~km} \mathrm{~s}^{-1}$. The line is an eye estimate of the best fit to the points. 
We therefore define this best fit as the ZAMS for A- and F-type stars, as a function of the parameter $\beta$. Furthermore, if the observed data for a given star, whether cluster member or field star, has a non-zero $\delta c_{1}$, we correct the ZAMS absolute magnitude for this 'evolutionary' effect. That is, $M_{v}=M_{V}$ (ZAMS) - $f \delta c_{1}$, where $f=8$ for an A star and $f=11$ for an $\mathrm{F}$ star.

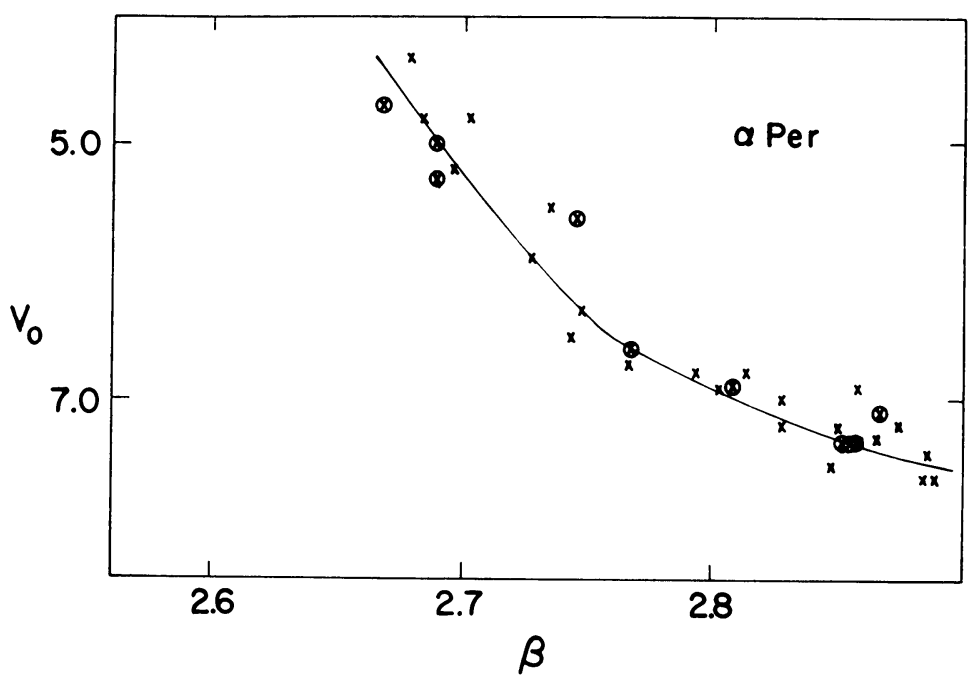

Fig. 13. As in Figure 12, but for members of the $\alpha$ Per cluster.

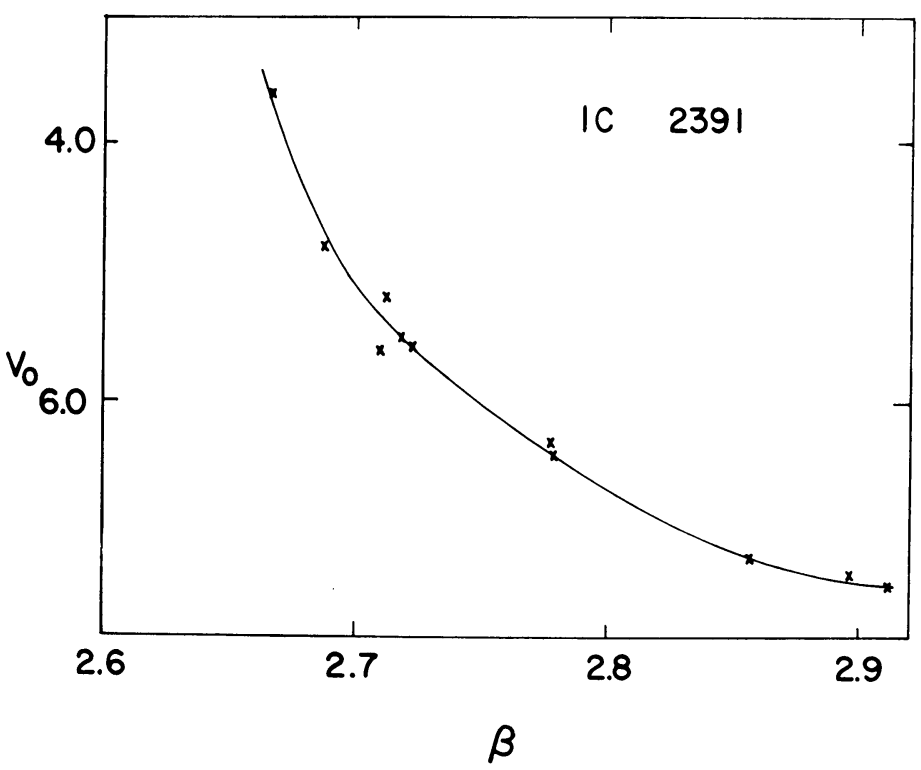

Fig. 14. The $V_{0}$ vs $\beta$ relation for B-type members of the cluster IC 2391. Data from Perry and Hill (1969). The line is an eye estimate of the best fit to the points. 
A 'by-product' of this preliminary calibration is the distance modulus to each of the clusters used in the fitting process. In particular, we find 5.5 for the Pleiades, 6.2 for the $\alpha$ Per cluster, and $7^{\mathrm{m}} .5$ for IC 4665 . We will use these values to fix the zero-point for the absolute magnitude calibration for the B-type stars.

(3) Determine the $V_{0}$ vs $\beta$ relation for clusters containing B-type stars. Data for each of the clusters referenced above were used. We show in Figures 12 to 16 the relations for several of the clusters: the Pleiades, $\alpha$ Per, IC 2391, Orion, and NGC 6231.

(4) Overlay these $V_{0}$ vs $\beta$ diagrams, sliding along lines of equal $\beta$, so as to determine the best fitting mean relation for all clusters. This procedure is quite similar to that used by Petrie for his $\mathrm{H} \gamma$ calibration. He had considerably less data to use, however. In doing this overlay, I was impressed that little evolutionary or $V \sin i$ effects appear to be present.

(5) Determine the zero-point for the resultant mean relation (that is, change the $V_{0}$ scale to $M_{V}$ by forcing the distance moduli of the three clusters of Step 2 to agree with the calibration). The resulting, preliminary, calibration is shown in Figure 17, as a smooth line. The symbols show Fernie's (1965) calibration, based on earlier, less complete, data.

The calibration also fits well the points for Sirius and Vega (from trigonometric parallaxes) and for Spica (from the interferometric work of Herbison-Evans et al. (1971)).

(6) Check the preliminary calibration for systematic errors to due age differences,

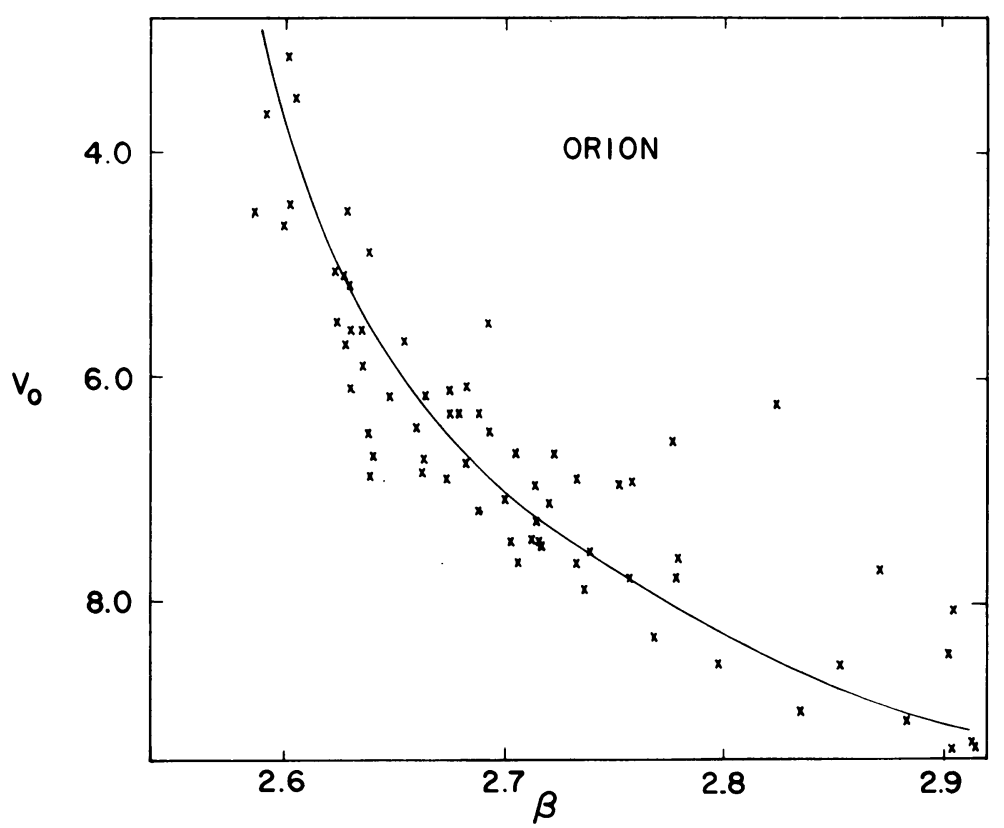

Fig. 15. The $V_{0}$ vs $\beta$ relation for B-type stars in the Orion association. The line is an eye estimate of the best fit to the points. 


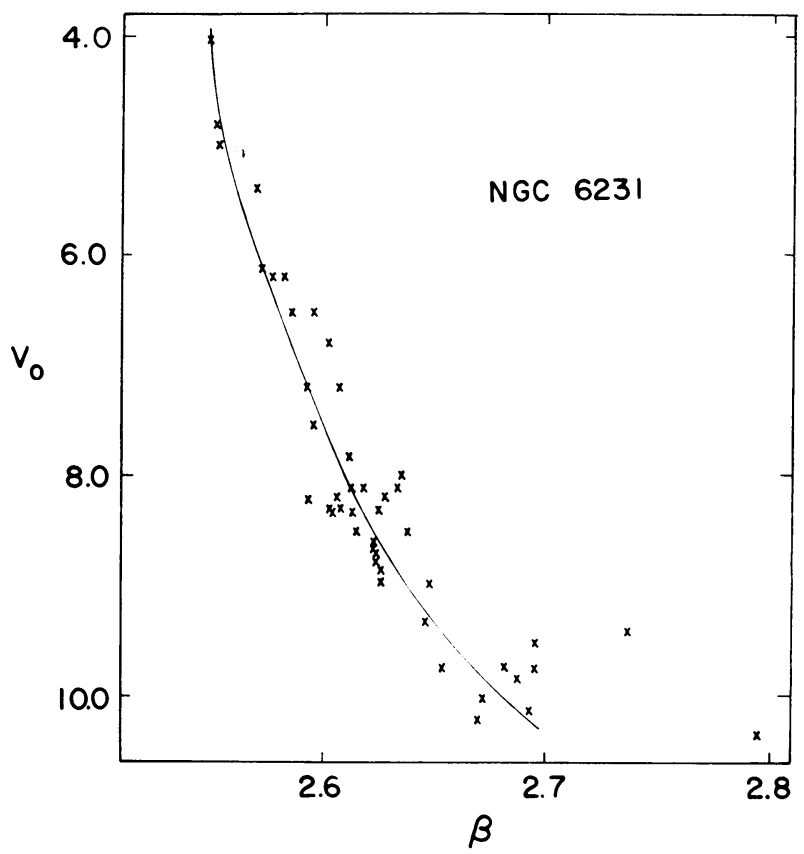

Fig. 16. As in Figure 15, but for members of the cluster NGC 6231.

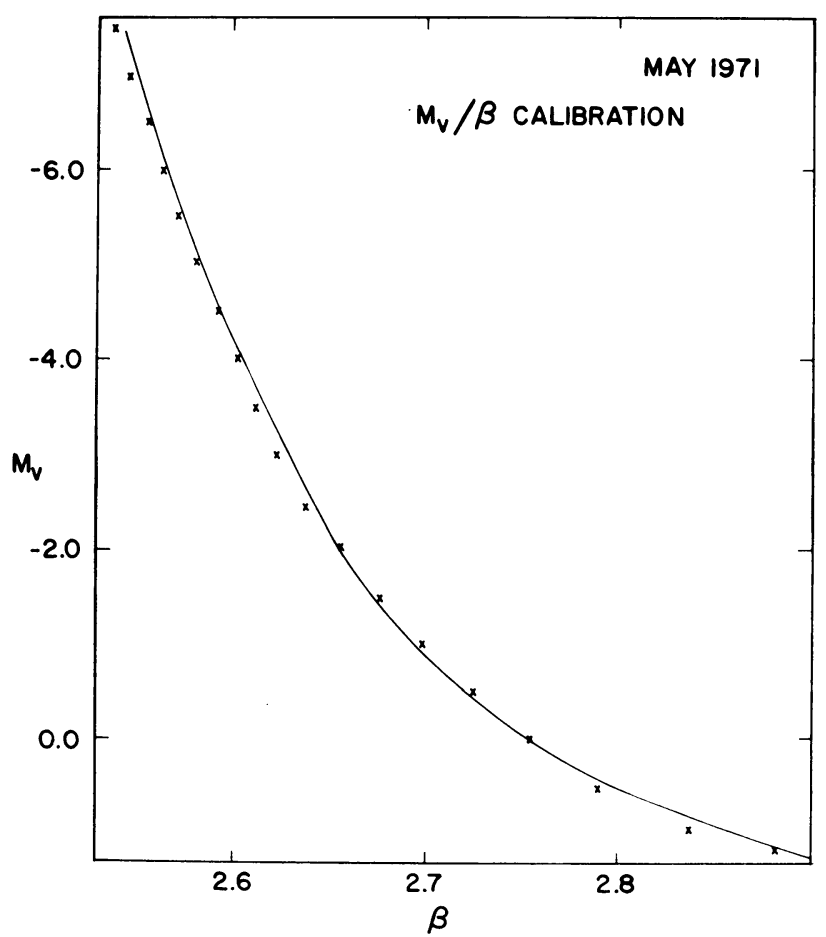

Fig. 17. The preliminary $M_{V}$ vs $\beta$ calibration. The crosses indicate the calibrations of Fernie (1965). The preliminary calibration is valid for stars on or near the ZAMS, but should be useful for evolved stars as well (see the text). 
rotational velocity effects, frequency-of-binary differences from cluster to cluster, spectral type effects, emission line stars, etc.

In general, we find few significant effects. In particular, there appear to be no systematic effects due to differences in $V \sin i$ from star to star, as Petrie (1965) also concluded from his photographic $\mathrm{H} \gamma$ work.

Small age effects, or spectral type effects, depending on your point of view, do exist, but they are less than in the cluster fitting techniques previously used, for example, by Johnson (1957) or Blaauw (1963).

The last two figures are propaganda for the hydrogen-line technique and calibration. Figure 18 shows the $\left(V_{0}-M_{V}\right)$ vs $V_{0}$ relation for the Pleiades, where evolutionary effects are certainly present. In the top part of the diagram, $M_{V}$ 's were determined using Blaauw's $M_{V}$ (ZAMS) vs $(U-B)_{0}$ calibration. In the bottom half, the $M_{V}$ 's were determined using the $M_{V}$ vs $\beta$ calibration of Figure 17. Figure 19 shows the equivalent diagram for the $\alpha$ Per cluster. Clearly the latter technique is to be preferred (at least, I think so!), especially for field stars.

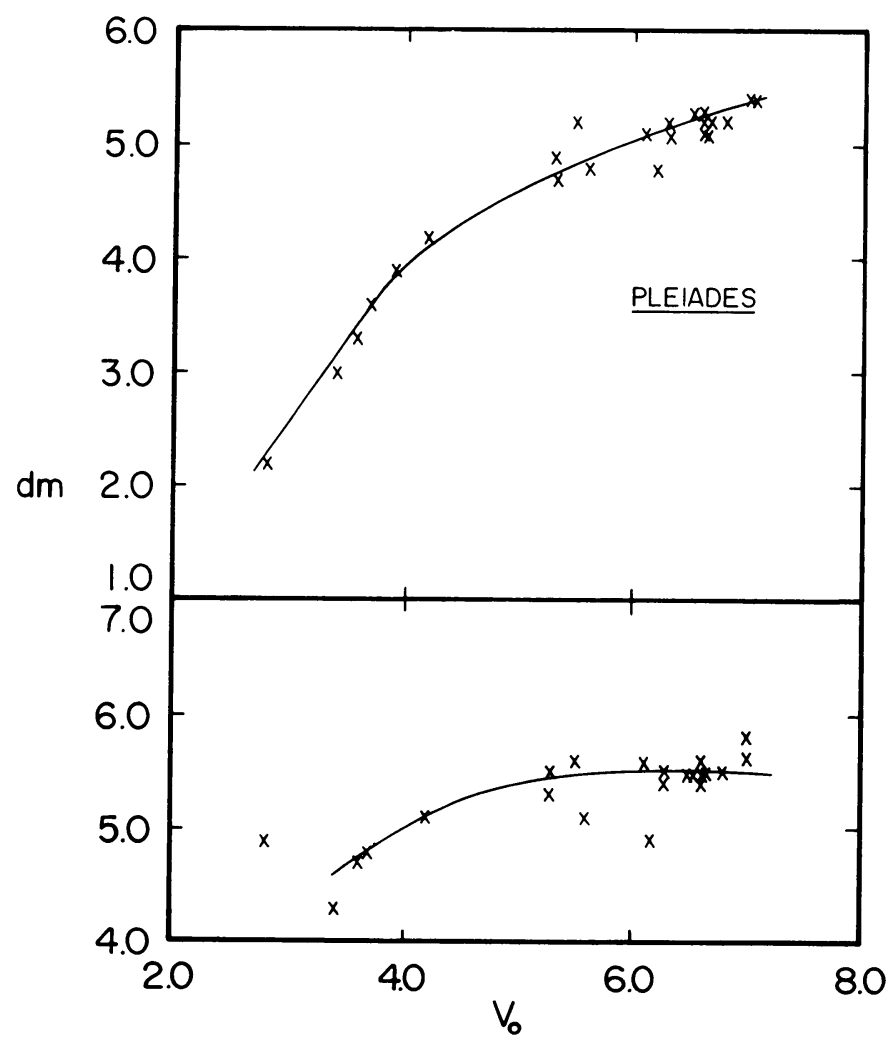

Fig. 18. The calculated distance modulus for B-type members of the Pleiades plotted versus their $V_{0}$ magnitude. Points on the top diagram were calculated using Blaauw's (1963) calibrations of $M_{V}$ (ZAMS) vs $(U-B)_{0}$; points in the bottom diagram using the calibrations shown in Figure 17. Curvature indicates 'evolutionary effects'. (see text). 
The final calibrations, nearly completed, will allow for these evolutionary effects via a $\delta M_{V}$ correction, in terms of a $\delta \beta$ above the ZAMS in the $\beta$ vs $c_{0}$ diagram.

Things remaining to be done before I am willing to label the calibration as final are:

(a) remaining checks for systematic effects,

(b) averages for each MK spectral type,

(c) final determination of the $\delta \beta$ factor as a function of spectral type, and

(d) comparisons to other absolute magnitude calibrations.

I would like to conclude by showing one of the comparisons; Table I summarizes the comparison. For stars of a given MK spectral type, I have determined the average

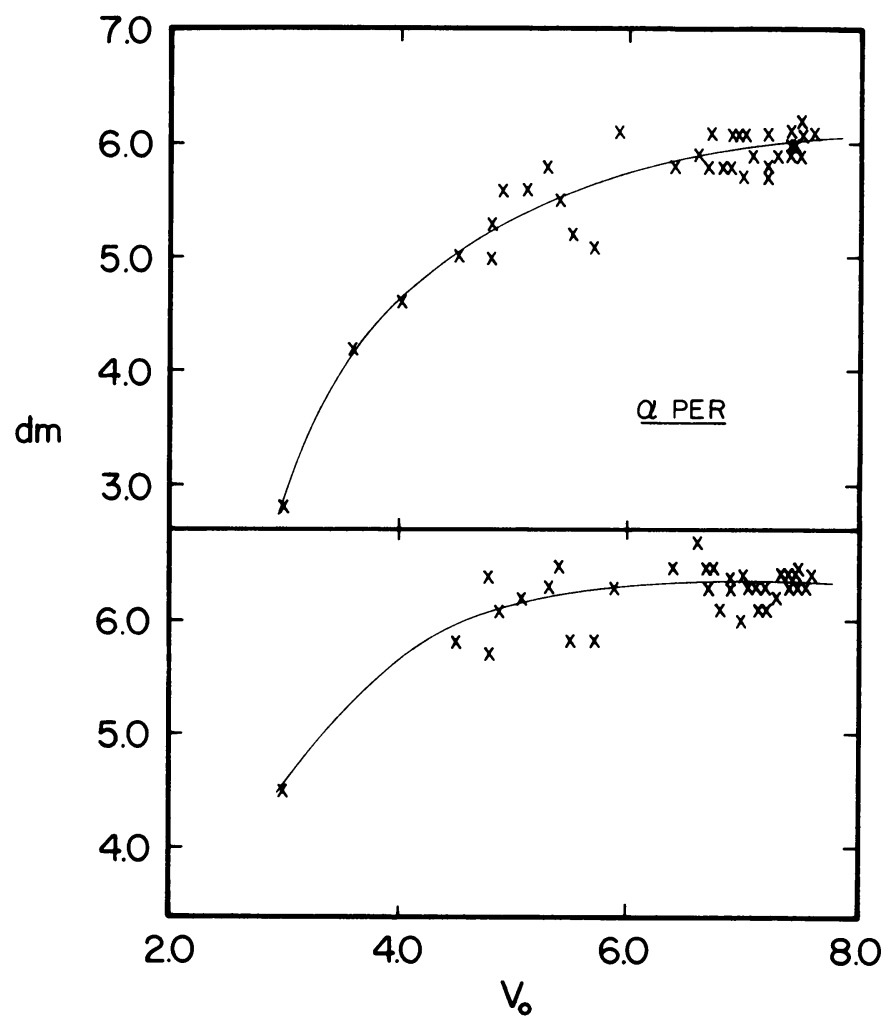

Fig. 19. As in Figure 18, but for members of the $\alpha$ Per cluster.

$c_{0}$ (there were about 20 stars in each sub-type). I then read off plots of $c_{0}$ vs $(U-B)_{0}$ the equivalent $(U-B)_{0}$ for each type. The resultant values agree closely with other author's average values for each sub-type, for example, Schmidt Kaler's (1965). I also read off the ZAMS line in the $c_{0}$ vs $\beta$ diagrams the value of $\beta$ equivalent to each average $c_{0}$. This $\beta$ then gives us a $M_{V}$ value from the calibration, valid for the ZAMS. From Blaauw's $M_{V}$ (ZAMS) vs $(U-B)_{0}$ calibrations, I also obtained an $M_{V}$ value valid for the ZAMS. Each of these values is given in Table I, and the agreement is excellent, better than I would have expected, perhaps. In any case, I think one can 
TABLE I

Comparison of the $\beta, M_{V}$ calibration with Blaauw's $(U-B)_{0}$, $M_{V}$ calibration, both valid for the ZAMS

\begin{tabular}{llllll} 
MK type & $c_{0}$ & $(U-B)_{0}$ & $\beta(Z A M S)$ & $M_{V}(\beta)$ & $M_{V}(U-B)_{0}$ \\
\hline O9 & -0.12 & -19.10 & $2 m 590$ & $-4 m 6$ & -4.5 \\
B0 & -0.07 & -1.05 & 2.608 & -3.9 & -3.9 \\
B1 & +0.02 & -0.96 & 2.629 & -2.9 & -2.8 \\
B2 & +0.15 & -0.84 & 2.658 & -1.9 & -1.8 \\
B3 & +0.33 & -0.67 & 2.701 & -1.0 & -0.9 \\
B4 & +0.37 & -0.63 & 2.709 & -0.8 & -0.8 \\
B5 & +0.42 & -0.59 & 2.720 & -0.6 & -0.6 \\
B6 & +0.48 & -0.55 & 2.735 & -0.3 & -0.3 \\
\hline
\end{tabular}

confidently use the $\left(\beta, M_{V}\right)$ calibration, especially for stars near the ZAMS, and, with care, even for evolved stars.

I hope to have the final calibration done and in press shortly (particularly if the 4-m telescope program goes smoothly this fall and winter!). I would be most happy to receive constructive criticisms both now and after the meeting, before I get the final calibration finished.

\section{Acknowledgments}

I wish to thank very much those who have allowed me to use their data in advance of publication. I also wish to thank Dr Bengt Strömgren for his encouragement and advice throughout all phases of the work, Mrs Jeannette Barnes, and Mr John Golson, without whom my research would have withered more than it has during my large telescope adventures, and those many astronomers with whom I have had many enjoyable and profitable discussions about photometry and calibrations.

\section{References}

Andrews, P. J.: 1968, Mem. Roy. Astron. Soc. 72, 35.

Anger, C. J.: 1931, Harvard Circ. No. 362.

Anger, C. J.: 1932, Haward Circ. No. 372.

Bappu, M. K. V., Chandra, S., Sanwall, N. B., and Sinvhal, S. D.: 1962, Monthly Notices Roy. Astron. Soc. 123, 521.

Beer, A.: 1961, Monthly Notices Roy. Astron. Soc. 123, 191.

Beer, A.: 1964, Monthly Notices Roy. Astron. Soc. 128, 261.

Blaauw, A.: 1963, Stars and Stellar Systems 3, 383.

Clariá, J. J.: 1971, Astron. J. 76, 639.

Cohen, H. L.: 1969, Astron. J. 74, 1168.

Cowley, A. P. and Crawford, D. L.: 1971, Publ. Astron. Soc. Pacific 83, 296.

Crawford, D. L.: 1958, Astrophys. J. 128, 185.

Crawford, D. L.: 1970, in A. Slettebak (ed.), Stellar Rotation, Reidel Publishing Co., Dordrecht, p. 204.

Crawford, D. L. and Barnes, J. V.: 1966, Astron. J. 71, 610.

Crawford, D. L. and Barnes, J. V.: 1969a, Astron. J. 74, 407.

Crawford, D. L. and Barnes, J. V.: 1969b, Astron. J. 74, 818.

Crawford, D. L. and Barnes, J. V.: 1970a, Astron. J. 75, 946.

Crawford, D. L. and Barnes, J. V.: 1970b, Astron. J. 75, 952. 
Crawford, D. L. and Barnes, J. V.: 1970c, Astron. J. 75, 978.

Crawford, D. L. and Barnes, J. V.: 1972, Astron. J. 77, 862.

Crawford, D. L., Barnes, J. V., Faure, B. Q., Golson, J. C., and Perry, C. L.: 1966, Astron. J. 71, 709.

Crawford, D. L., Barnes, J. V., Gibson, J., Golson, J. C., Perry, C. L., and Crawford, M. L.: 1972, Astron. Astrophys. Suppl. 5, 109.

Crawford, D. L., Barnes, J. V., and Golson, J. C.: 1970, Astron. J. 75, 624.

Crawford, D. L., Barnes, J. V., and Golson, J. C.: 1971a, Astron. J. 76, 621.

Crawford, D. L., Barnes, J. V., and Golson, J. C.: 1971b, Astron. J. 76, 1058.

Crawford, D. L., Barnes, J. V., Hill, G., and Perry, C. L.: 1971, Astron. J. 76, 1048.

Crawford, D. L., Glaspey, J. W., and Perry, C. L.: 1970, Astron. J. 75, 822.

Crawford, D. L. and Mander, J.: 1966, Astron. J. 71, 114.

Crawford, D. L. and Perry, C. L.: 1966, Astron. J. 71, 206.

Fernie, J. D.: 1965, Astron. J. 70, 575.

FitzGerald, M. P.: 1970, Astron. Astrophys. 4, 234.

Furenlid, I.: 1971, Astron. Astrophys. 10, 321.

Garrison, R. F.: 1967, Astrophys. J. 147, 1003.

Glaspey, J. W.: 1971, Astron. J. 76, 1041.

Graham, J. A.: 1967, Monthly Notices Roy. Astron. Soc. 135, 377.

Hack, M.: 1953, Ann. Astrophys. 16, 417.

Hardie, R. H. and Crawford, D. L.: 1961, Astrophys. J. 133, 843.

Herbison-Evans, R., Hanbury Brown, R., Davis, J., and Allen, L. R.: 1971, Monthly Notices Roy. Astron. Soc. 151, 161.

Hill, G. and Perry, C. L.: 1969, Astron. J. 74, 1011.

Hoag, A. A. and Applequist, N. L.: 1965, Astrophys. J. Suppl. 12, 215.

Jenkins, L. F.: 1963, General Catalogue of Trigonometric Stellar Parallaxes, Yale University Observatory, New Haven.

Johnson, H. L.: 1957, Astrophys. J. 126, 121.

Johnson, H. L. and Iriarte, B.: 1958, Lowell Obs. Bull. 4, 47.

Johnson, H. L. and Morgan, W. W.: 1953, Astrophys. J. 117, 313.

Kopylov, I. M.: 1958, Izv. Krymsk. Astrofiz. Obs. 20, 156.

Lindblad, B.: 1922, Astrophys. J. 55, 85.

Lindblad, B.: 1925, Nova Acta Rej. Soc. Sci. Upsala, Ser. 4, 6, No. 5.

Lindblad, B.: 1926, Medd. Astr. Obs. Uppsala, No. 11.

Mihalas, D.: 1972, Astrophys. J. 176, 139.

Öhman, Y.: 1935, Stockholm Obs. Ann. 12, No. 1.

Perry, C. L. and Hill, G.: 1969, Astron. J. 74, 899.

Petrie, R. M.: 1950, Publ. Dominion Astrophys. Obs. 8, 319.

Petrie, R. M.: 1953, Publ. Dominion Astrophys. Obs. 9, 251.

Petrie, R. M.: 1956, Vistas in Astronomy 2, 1346.

Petrie, R. M.: 1965, Publ. Dominion Astrophys. Obs. 12, 317.

Petrie, R. M. and Maunsell, C. D.: 1950, Publ. Dominion Astrophys. Obs. 8, 253.

Petrie, R. M. and Moyls, B. N.: 1956, Publ. Dominion Astrophys. Obs. 10, 287.

Schmidt-Kaler, Th.: 1965, in K. H. Hellweg (ed.), Landolt-Börnstein, Springer-Verlag, Berlin and New York, New Ser., Group 6, Vol. 1, p. 284.

Sinnerstad, U.: 1954, Stockholm Obs. Medd., No. 82.

Sinnerstad, U.: 1961a, Stockholm Obs. Ann. 21, No. 6.

Sinnerstad, U.: 1961 b, Stockholm Obs. Ann. 22, No. 2.

Strom, K. M., Strom, S. E., and Yost, J.: 1971, Astrophys. J. 165, 479.

Strömgren, B.: 1951, Astron. J. 56, 142.

Strömgren, B.: 1952, Astron. J. 57, 200.

Strömgren, B.: 1956a, in J. Neyman (ed.), Proceedings of the Third Berkeley Symposium, University of California Press, Berkeley and Los Angeles, Vol. 3, p. 49.

Strömgren, B.: 1956b, Vistas in Astronomy 2, 1336.

Strömgren, B.: 1958, in D. J. K. O'Connell (ed.), Stellar Populations, Interscience Publishers, New York, p. 385.

Strömgren, B.: 1963, Stars and Stellar Systems 3, 123.

Strömgren, B.: 1966, Ann. Rev. Astron. Astrophys. 4, 433. 
Strömgren, B. and Perry, C.: 1965, unpublished report, Institute for Advanced Study, Princeton, N. J.

Williams, E. G.: 1936, Astrophys. J. 83, 279.

\section{DISCUSSION}

Crampton: In the case of known binary stars did you make correction for duplicity?

Crawford: We did not make any correction for duplicity. The binaries are generally included in the discussion.

Blaauw: How large would you estimate the probable error of the main sequence fit to the trigonometric parallax stars, i.e., the p.e. of the zero point of the newly derived $M_{V}$ system?

Crawford: Something like $0 \mathrm{~m} 1$ or $0 \mathrm{~m} 2$.

Wesselink: Could you use your $M_{V}$ vs $\beta$ technique to the non emission (apparently fainter) B stars in the Magellanic Clouds with consequent result for the distance modulus?

Crawford: Yes, we are observing just such stars at the present time.

Schmidt-Kaler: You showed a diagram $\mathrm{H} \alpha$ vs $\mathrm{H} \beta$ with quite a few emission B stars, and a diagram $\mathrm{H} \beta$ vs $\mathrm{H} \gamma$ with very few. Did you put the same stars in both diagrams? Or does this mean that you find a discontinuous Balmer jump?

Crawford: Most of the stars are the same. Many more stars show emission at $\mathrm{H} \alpha$ than at $\mathrm{H} \beta$ or $\mathrm{H} \gamma$.

Jaschek: Did you observe in Orion stars which show helium line anomalies? Did you exclude B-type peculiar stars or, in general, peculiar stars?

Crawford: We observed a few of the stars in Orion that you refer to. Some look odd in our photometry, some do not. In general, we include peculiar stars in our work. The Am stars fit the calibrations for the A stars quite well. Most Ap stars look like B stars to me.

Maeder: With regard to the position of the Of stars in some of your diagrams one should note than, according to Walborn, the f-characteristics may be identified with a luminosity effect. One may show that the different position of the Of stars in $(U-B)$ vs $(B-V)$ diagram is in complete agreement with the luminosity effect predicted by the recent non-LTE models of Auer and Mihalas. This may be considered as a supplementary support to the hypothesis that the Of stars are intrinsically brighter that the so-called normal O-type stars. The observed difference is not due to the contribution of the emission lines in the filters $(<0$ m.003) but to a change produced by the luminosity effect in the energy distribution.

Blaauw: Do stars in the Taurus stream, which is associated with the Hyades, behave similar to the Hyades proper in the $c_{1}$ vs $\beta$ diagram?

Crawford: Yes, most of them look like stars in the Hyades cluster, according to Eggen.

Jones: I find that a back-warming correction is required in $\beta$ when comparing stars of very different metal abundance. It amounts to roughly $0 \mathrm{~m} .05$ between stars of 0.01 the solar abundance and those of solar abundance.

Murray: Could the Hyades discrepancy be accounted for by slight differences in the proper motion system depending on apparent magnitude, leading to systematic differences in the absolute magnitudes, also depending on apparent magnitude?

Crawford: I don't think so; if anything, the photometry would indicate that there are no such difficulties.

Garrison: Isn't it disturbing that the two clusters which you find do not fit are the only old, rich clusters?

Crawford: Yes, however, the statistics of small numbers allow all sorts of puzzles.

Hauck: I just want to mention that all published measurements in the uvby $\beta$ system have been compiled here by Lindemann and myself. It is possible to obtain the tape at the Centre de Données Stellaires in Strasbourg. Now we have 6000 stars and we hope to publish as soon as possible a general catalogue with homogeneous data. 